\title{
Digital Scores Galore: nkoda Digital Sheet Music Library
}

\section{Reviewed by Trevor Deck, University of Toronto}

The publishing market for digital sheet music lags significantly behind its counterparts in the ebook and digital media world. Most music libraries still dedicate the majority of their music score budgets to physical acquisitions. The abrupt closure of libraries in March 2020, as a result of the COVID-19 pandemic, highlighted the need for increased digital access to sheet music. The University of Toronto spent the summer evaluating different digital sheet music platforms in preparation for a largely online learning environment in the fall. One relatively new UK-based company, nkoda, stood out as a product with the potential to shift the primary means by which we access sheet music.

The past decade has seen a fundamental change in the way people consume books, recorded music, and film. While commercial markets for these products are different from that of sheet music, the increasingly common practice of using mobile devices as a primary means of consuming media has led musicians to adopt tablets as a means of accessing printed music. By offering access to over 80,000 scores from over 100 publishers, including Boosey \& Hawkes, Bärenreiter, Breitkopf \& Härtel, and Ricordi, nkoda has created a platform that has the potential to convert many more musicians to digital music collections. While there are a number of options for digital scores in the academic library market (Alexander Street Press' "Music Online: Classical Scores Library" or the contemporary e-score platform "Babelscores," for instance), most of these platforms contain a relatively dated interface and feature materials from a limited number of publishers, with little to no new content added. In contrast, nkoda offers a modern user interface and access to an unprecedently rich and continuously expanding collection of scores from many of the world's leading sheet music publishers (see Figure 1).

Of the more notable publishers on the platform, Ricordi's coverage is the most comprehensive, with over 8,700 scores available as of May 2020, based on statistics provided by nkoda. Boosey \& Hawkes is also very well represented, with over 5,000 scores available. While the quantity of scores available from other prominent publishers may appear less remarkable, the quality of scores found in nkoda from publishers such as Bärenreiter is still noteworthy. For instance, the platform contains the newly published Bärenreiter Urtext edition of Beethoven's complete piano sonatas and symphonies. One obvious gap is Henle, which just recently began offering institutional subscriptions to its scores via its own digital platform.

The variety in terms of types of scores available on nkoda is similarly comprehensive. Table 1 provides a quantitative breakdown of the different types of scores available. It should be noted that there is some overlap in these categories. For example, "guitar" represents all the guitar music on the platform, not just solo guitar music. The large selection of full scores and parts is particularly impressive. The downside is that these are not licensed for performance rights. (More on this aspect below.)

\section{(i) (5)


Figure 1: Number of scores by publisher (as of May 2020)

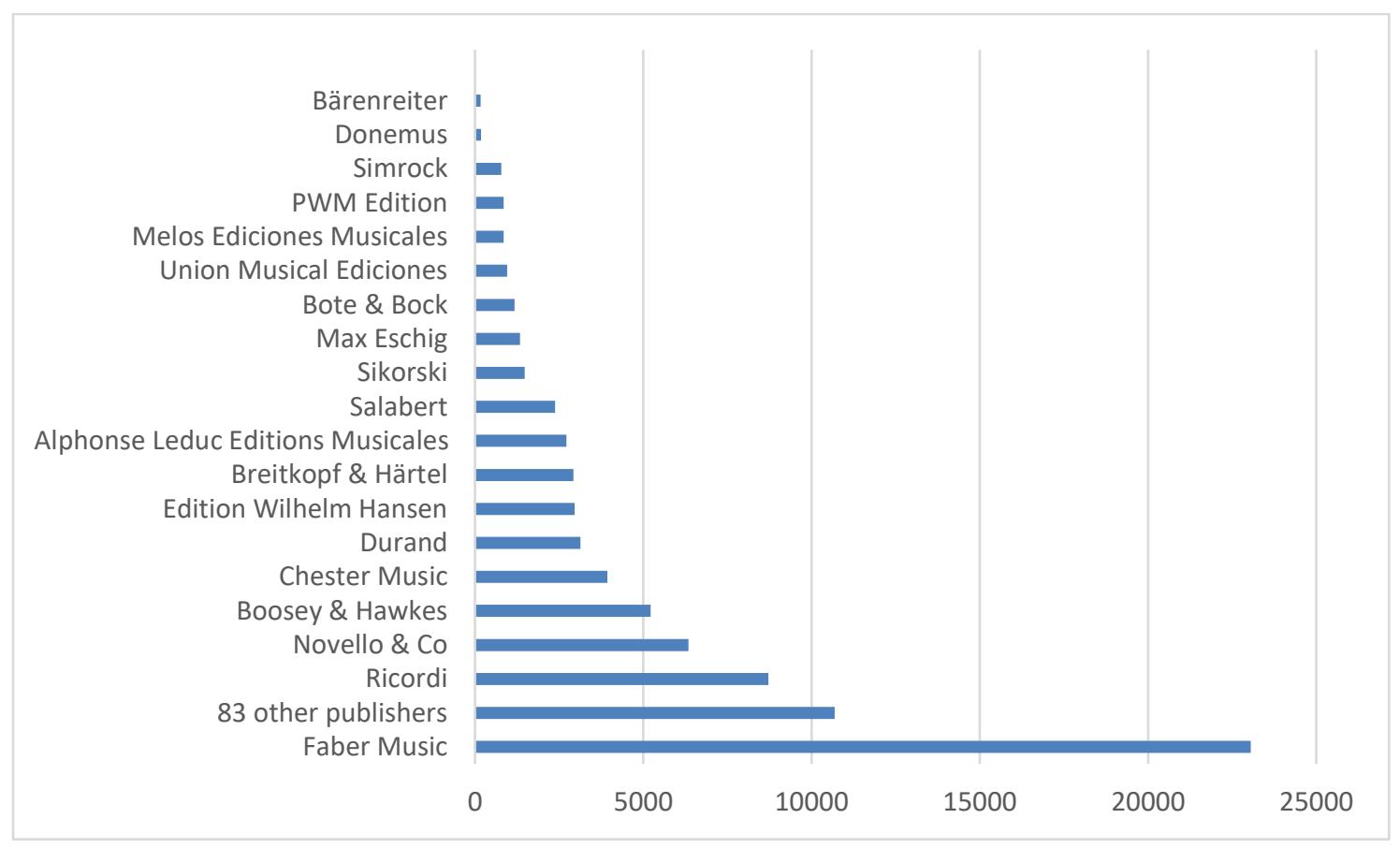

Table 1: Collection breakdown by instrumentation (as of May 2020)

\begin{tabular}{|l|l|}
\hline Type of score & Number of scores \\
\hline Piano & 32,757 \\
Voice & 21,099 \\
Guitar & 15,066 \\
Full Score & 9478 \\
Ensemble & 4155 \\
Mixed Chorus SATB & 3240 \\
Strings & 2176 \\
Vocal Score & 2028 \\
Piano Reduction & 1680 \\
String Quartet & 1375 \\
Saxophone & 1147 \\
Brass & 779 \\
Full Score Plus Parts & 704 \\
Opera Vocal Score & 574 \\
Study Score & 526 \\
Chorus Score & 387 \\
Opera Score & 347 \\
Playing Score & 79 \\
\hline Total number of scores & 80,001 \\
\hline
\end{tabular}


When first signing up for an account in nkoda, the app provides a series of options to help personalize the experience (Figure 2). This allows the app to function in much the same way as commercial music and video streaming services, curating a personal experience for users by allowing them to discover new music based on their preferred instruments, genres, level of difficulty, and ensemble size. The homepage also takes a lot of cues from apps such as Netflix and Spotify, providing the user with a scrolling list of suggestions based on their preferences and curated "playlists" based on specific themes (Figures 3 and 4).

The emphasis on personally curated discovery in the nkoda interface provides a new way to explore sheet music collections. However, some users may be frustrated by the lack of search filters and options. The interface is currently limited to searching by "artist" or "title" only, though searches in the title field for publisher, genre, and instrumentation keywords seem to produce relevant search results. There is no way to refine one's results after an initial search. Moreover, the search function does not appear to recognize multiple languages in search results. For instance, searching for "concerto" will not bring up results for "Konzert," nor does it seem capable of identifying multiple spellings of transliterated names (i.e. Rachmaninov/Rachmaninoff). The lack of search and filter abilities represents one of the major weaknesses of the app.

Figure 2: Personalized options

You play

III Librarian $\times$

Medium difficulty. Alone and Small group and Teacher. Edu...

Electric Guitar $\times$ Medium difficulty. Alone and Small group. Educational, Jazz...

溲: Guitar $\times$ Medium difficulty. Alone and Small group and Teacher. Edu...

Add another instrument

Q Search for an instrument

\begin{tabular}{|c|c|c|c|c|}
\hline W] & . & 据 & & $d y$ \\
\hline Piano & Soprano & Violin & Flute & Conductor \\
\hline W & 13 & & & $f$ \\
\hline
\end{tabular}

\section{I'm done $\checkmark$}


Figure 3: Home screen with suggested repertoire based on personalized settings

-

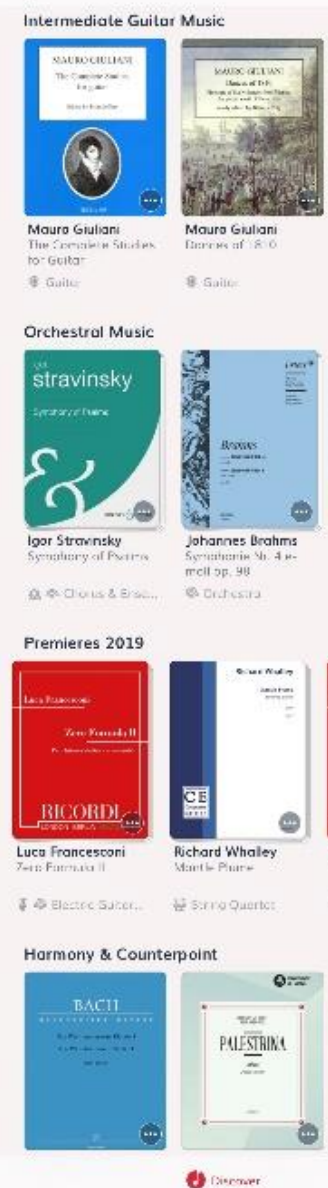

Onkoda
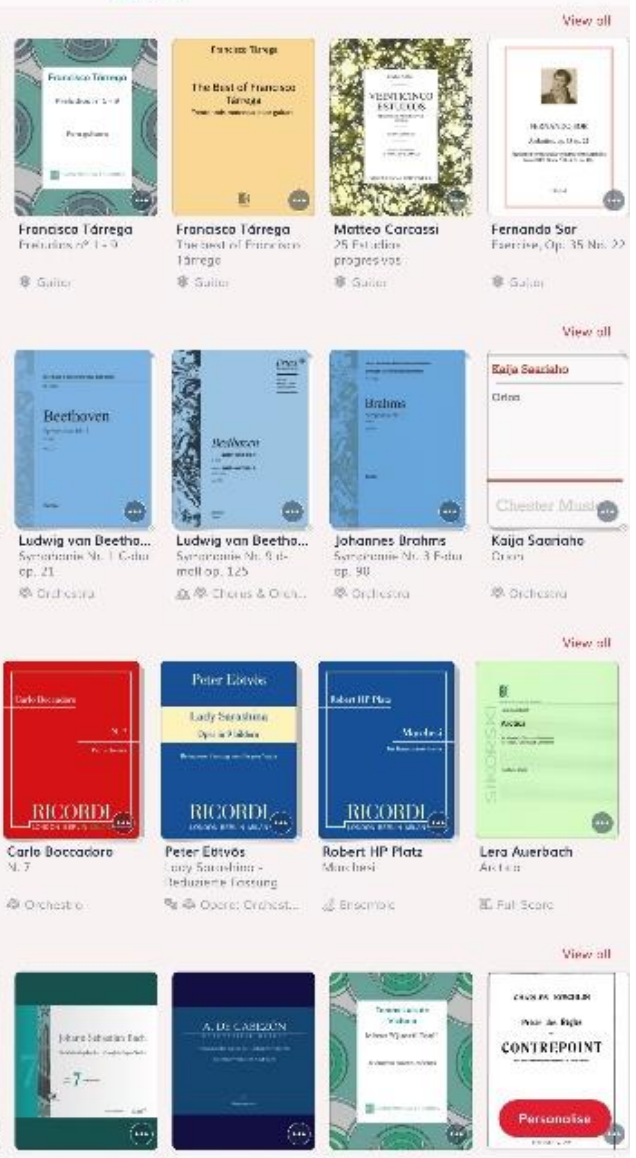

Q serret

Figure 4: Playlists
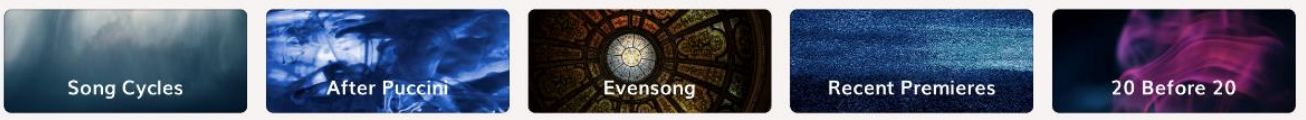

Now That's What I Call Microtonality

nkoda has been designed to mimic the consumer experience in commercial streaming culture. This is evident in some of the terminology contained within the app (for example, the use of "artist" as opposed to "composer" in the search field) as well as in the user experience, which prioritizes discovery through browsing rather than through searching and filtering. Unfortunately, in creating this user experience, considerations of discoverability from a cataloguing and classification perspective have been largely ignored. MARC records are not currently available, although the vendor understands the importance this feature would hold for the library community. Another limitation of the platform is the restriction of content access to within the nkoda app. While users may download scores within the app for offline use, they cannot print them. This decision was likely made to quell publishers' concerns around copyright infringement. Still, the inability to access scores from a browser inherently limits the degree to which scores can be discovered and accessed from within a library catalogue, even if MARC 
records are provided in the future. These challenges are understandable, as the vendor is new to the academic library market. However, for nkoda to gain widespread adoption within the music library community, it will need to provide a product that has library models of access in mind. nkoda is making some progress in this respect. For example, setting up the app was particularly complicated and impractical for large institutions throughout the first few years of development, as they catered almost exclusively to individual subscribers. Now the app offers Shibboleth authentication. While it remains necessary for new users to create personal accounts in order to access content, they can connect to the resource with relative ease using their institutional login credentials. Further attention to library needs ought to be a priority for nkoda as they continue to develop their platform.

Feedback regarding the contents of the collection from students and faculty at the University of Toronto throughout a trial of nkoda was mostly positive. With respect to classical music, the platform offers an unprecedentedly large collection of digital scores that is rivalled only by IMSLP. Given its inclusion of modern editions from many renowned publishers, nkoda really is breaking new ground in how musicians can access and engage with sheet music not yet in the public domain. There is also a substantial amount of pedagogical material, making the platform a useful tool for students of all ages as well as music educators. Although nkoda boasts an unrivalled selection of classical and contemporary instrumental music, its offerings are unlikely to satisfy everyone. Jazz coverage, for example, is spotty and leaves a lot to be desired. While there is strong coverage of certain composers (e.g., 213 scores by Cole Porter), searching for transcriptions of Miles Davis brings up only a single result. In terms of representation by nationality (see Figure 5), American and British composers dominate, numbering approximately 800 each. There are 60 Canadian composers in total, many of whom are more commonly known as popular recording artists than composers (see Table 2).

Figure 5: Number of composers by nationality (as of May 2020)

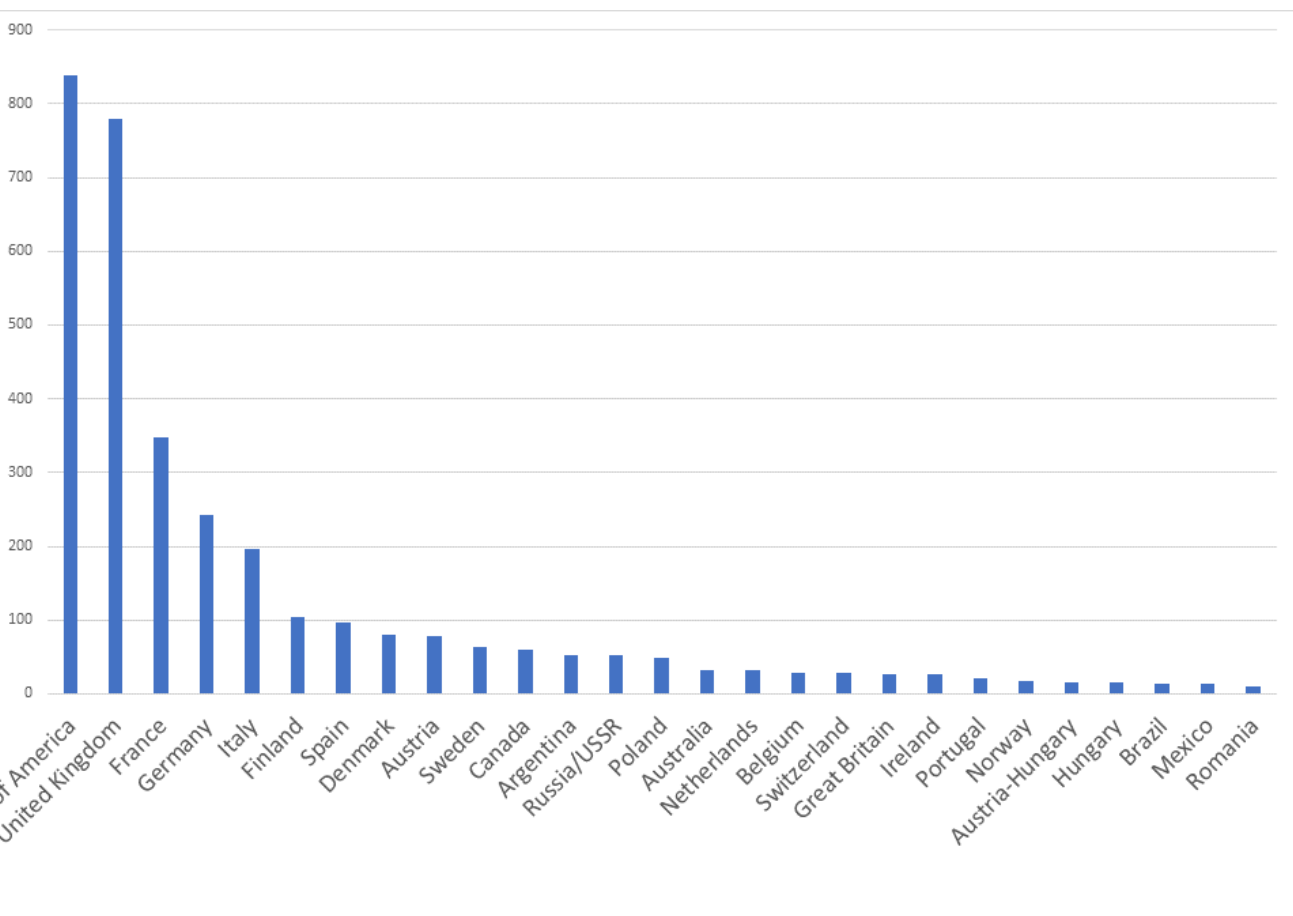


Table 2: Ranking of Canadian composers by number of scores in nkoda (as of May 2020)

\begin{tabular}{|l|c|}
\hline Composer & \# of Scores \\
\hline Bublé, Michael & 64 \\
Vivier, Claude & 38 \\
Doolittle, Emily & 33 \\
Farnon, Robert & 21 \\
Chatman, Stephen & 15 \\
Cohen, Leonard & 15 \\
Adams, Bryan & 12 \\
Dion, Celine & 11 \\
Krall, Diana & 11 \\
Moussa, Samy & 9 \\
Bieber, Justin & 8 \\
Lee, Geddy & 8 \\
MacDermot, Galt & 7 \\
Allen, Peter & 6 \\
Holman, Derek & 6 \\
Kroeger, Chad & 6 \\
Whittall, Matthew & 6 \\
Faith, Percy & 5 \\
Foster, David & 5 \\
Schafer, R. Murray & 5 \\
Butler, Will & 4 \\
Fox, George & 4 \\
Furtado, Nelly & 4 \\
Lavigne, Avril & 4 \\
Powter, Daniel & 4 \\
Seitz, Ernest & 4 \\
Shore, Howard & 3 \\
Tremblay, Gilles & \\
Fuller, Jerry & \\
Mitchell, Joni & \\
29 more with 1-2 scores each & \\
\hline
\end{tabular}

It is worth noting that one can search the nkoda library from the company's homepage without logging in, so that those who are considering a subscription can evaluate the score collection first. The company also currently offers a free seven-day trial for individual subscribers (credit card required) as well as limited free trials for institutions.

While the limited searching and browsing functionalities leave a lot to be desired from a user interface perspective, the functionality of the app when it comes to reading and marking up music is excellent. Scores load quite quickly and appear crisp and easily legible on a 12.9-inch iPad Pro. I would highly recommend a screen this size if intending to fully embrace digital sheet music. Tapping the bottom left 
or right of the screen allows the user to easily turn pages forward or backward. There is also an option to scan through the piece in a scrolling view, which comes in handy when reading long scores.

The app also features Bluetooth foot pedal integration to allow for hands-free page turning. The overall user experience when interacting with scores is very intuitive and features an abundance of options for annotating pieces. There are hundreds of pre-set symbols to choose from, as well as the ability to pencil in free text and highlight sections (Figure 6). Perhaps my favourite feature is the ability to save multiple annotations for the same score, so a musician can have one copy for practice, perhaps a cleaner version for performance, and yet another for teaching purposes. The app also allows the user to save scores for offline access and to upload one's own scores. Musicians can even share annotations and scores with other nkoda users, which offers some wonderful possibilities when teaching and performing in ensembles.

\section{Figure 6: Annotations}

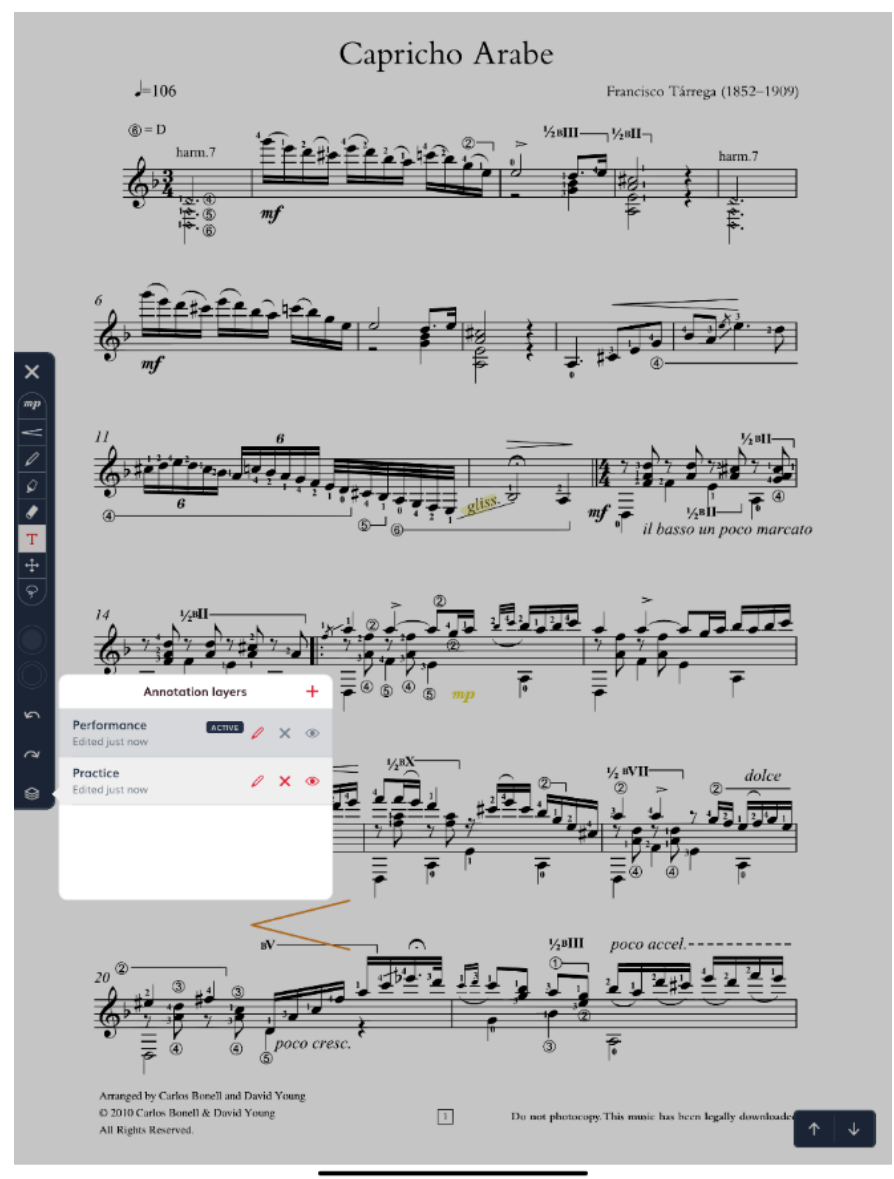

Finally, a discussion of a resource of this type from a library perspective would not be complete without considering the licensing model and financial implications of the service. Despite its impressive collection, the temporary nature of nkoda's subscription-based licensing model is unlikely to convince libraries to stop collecting physical scores. Unless nkoda decides to shift its business model to one that allows for perpetual licensing of individual scores, the platform can only serve as a supplement to print 
collections rather than a replacement. Quotes for institutional licensing were significantly more expensive than other digital score platforms on the market. This is not surprising given the noteworthy array of publishers involved and the inclusion of many newly published editions. The expense, however, may be a barrier to access for many institutions. nkoda does offer graduated pricing based on the number of simultaneous users licensed (options include 10,15,20, or 150), but the costs are significant at every level. It is also worth noting that despite an option for 150 simultaneous users (what nkoda calls the "unlimited user option") and the availability of a substantial collection of complete scores, nkoda licenses do not include performance rights. Musicians wishing to perform from nkoda's online scores will therefore need to contact publishers to request public performance rights for any scores they wish to perform in public. To this end, nkoda has partnered with a company called Zinfonia to provide a streamlined means of requesting performance rights for the full scores available on the app. When viewing a record for a full score, a disclaimer appears at the top of the screen with a link to contact the publisher through Zinfonia (Figure 7).

Figure 7: Performance rights disclaimer and link to request performance rights through Zinfonia

\section{zin onia mogervususter}

home search my account for publishers help

\section{Request Materials}

Vivier, Claude

for soprano, wind quintet, string quintet and tape

[Boosey \& Hawkes (Hendon)]

Request a performance agreement and/or parts from a publisher/distributor

You will be now asked a series of questions to process your request.

You can resume an incomplete order at any time by clicking the same link you used to get to this page

No payments are required at this stage. Once your request has been accepted by the distributor, they will be in touch with details about their requirements to fulfil the

order.

Note: The order is not submitted until all steps have been completed, and it is important to complete All sections answering all questions to avoid any delay to your order.

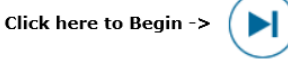

While nkoda is unlikely to convince music libraries to stop collecting physical music scores, it does have the potential to be a gamechanger in the wholesale acceptance of digital scores. If the platform is to gain widespread adoption in libraries, it will need to focus more on creating a platform designed with libraries and library users in mind. With some improvements in accessibility and discoverability, as well as a continued dedication to building the most comprehensive digital collection of copyright-protected scores on the market, nkoda has serious potential to bring the sheet music industry into the twenty-first century. 